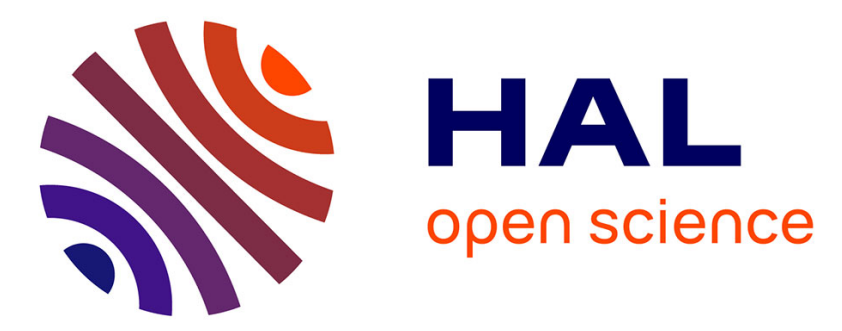

\title{
Potential Game approach to virus attacks in network with general topology
}

François-Xavier Legenvre, Yezekael Hayel, Eitan Altman

\section{To cite this version:}

François-Xavier Legenvre, Yezekael Hayel, Eitan Altman. Potential Game approach to virus attacks in network with general topology. NETGCOOP 2016 - International conference on NEtwork Games. Optimization and Control, Nov 2016, Avignon, France. hal-01378846

\section{HAL Id: hal-01378846 \\ https://hal.inria.fr/hal-01378846}

Submitted on 10 Oct 2016

HAL is a multi-disciplinary open access archive for the deposit and dissemination of scientific research documents, whether they are published or not. The documents may come from teaching and research institutions in France or abroad, or from public or private research centers.
L'archive ouverte pluridisciplinaire HAL, est destinée au dépôt et à la diffusion de documents scientifiques de niveau recherche, publiés ou non, émanant des établissements d'enseignement et de recherche français ou étrangers, des laboratoires publics ou privés. 


\title{
Potential Game approach to virus attacks in network with general topology
}

\author{
François-Xavier Legenvre, Yezekael Hayel’ and Eitan Altman ${ }^{\ddagger}$
}

\begin{abstract}
SIS epidemic non-zero sum games have been recently used to analyse virus protection in networks. A potential game approach was proposed for solving the game for the case of a fully connected network. In this paper we manage to extend this result to an arbitrary topology by showing that the general topology game is a potential game. We apply this result to study numerically some examples.
\end{abstract}

\section{Introduction}

Computer viruses have been reported to cause damage of 17 billion US\$ in 2000 . Already in 1998, the relation between computer viruses and epidemiology are suggested [9]. Since then, viruses and tools to fight them have become more sophisticated. Nowadays cyber security is not only defensive and are used as warfare also, see [5].

In the biology literature, no references to games in epidemics are published before 2000. Some research works on the topic have appeared only recently [1, 3, 2]. A natural modeling of how to fight viruses is through the use of zero-sum games [6]. A big boost to epidemics modelling followed the work of P. Van Mieghem and coauthors in several papers [7, 12]. In these papers, the authors provide bounds on the dynamics of SIS epidemic models as well as meanfield approximations on their metastable regime.

Game models (static ones) based on the above SIS models appear already in 2009 [10] and later at [4]. In these models the virus is not assumed to be strategic as the authors focus on the study of non-cooperative strategic network nodes. Each of these nodes is faced with the problem of whether or not to purchass a vaccination (or anti-virus). In this paper, we revisit the model of [4] and extend it in two ways: we consider a general topology (where as [4] considers a fully connected network), and we allow for arbitrary node dependent infection and healing rates.

\footnotetext{
${ }^{*}$ CERI/LIA, University of Avignon, France, email: francois-xavier.legenvre@univ-avignon.fr

†CERI/LIA, University of Avignon, France email: yezekael.hayel@univ-avignon.fr

${ }^{\ddagger}$ Côte d’Azur University, INRIA, France, and LINCS, Paris, France, email: eitan.altman@inria.fr
} 
In [4] the authors solve the game and establish existence of pure equilibria by showing that it is equivalent to a congestion game (in the sense of Rosenthal) which is known to be a potential game and for which explicit expressions of the potential exist. The fact that the network is fully connected is used in order to ensure that the cost of a defense action by a node only depends on how many other nodes use the same action, which is one of the assumptions in the congestion game formalism.

In this paper, we manage to show that the virus protection game between the nodes of the network has a potential for an arbitrary topology and therefore a pure equilibrium exists. We note that this result is not a direct application or Rosenthal's congestion game as the infection quasi stable probability of a node, say $i$, depends in a complex way on the network topology and not just through the number of other nodes that take the same action as $i$. We finally introduce an algorithm to solve the game and apply it to some examples.

\section{Model}

We set a graph called $\mathscr{N}(N)$ with $N$ the number of nodes representing our network. There exists an adjacency matrix $A$, representing by the coefficients $A_{i, j}$, which is worth 1 if there exists a link between the node $i$ and the node $j$. As aforementioned, each node is singular, which means that all nodes have different recovery and infection rates providing us a general framework for epidemics. Several epidemic dynamics are proposed in the literature but the Susceptible-Infected-Susceptible (SIS model) is one of the most studied and can be described as follows. An infected node $i$ contaminates each of his healthy neighbor with rate $\beta_{i}$, and the remaining time for which the node $i$ is infected follows an exponential process with rate $\delta_{i}$. After being recovered from the infection, each healthy node becomes susceptible again, and can be again infected. Considering an SIS epidemics over a graph, there exists a limiting spreading factor rate, denoted by the critical epidemic threshold, below which the infection vanishes exponentially fast in time, and above which the critical threshold the network stays infected. Each individual $j$ decides to be protected (i.e. $\left.\sigma_{j}=0\right)$ or not $\left(\sigma_{j}=1\right)$. Therefore, we consider action-dependent SIS dynamics because the infection rate of node $i$ depends on his action $\sigma_{i}$, that is the infection rate of node $i$ is given by $\beta_{\sigma_{j}}^{j}$.

With the aforementioned network structure and epidemic dynamics, the dynamics of the probability $V_{i}(t)$ for node $i$ to be infected by the virus at time $t$, can be approximated, thanks to the NIMFA equation [13], as follows:

$$
\frac{d V_{i}(t)}{d t}=-\delta_{i} V_{i}(t)+\left(1-V_{i}(t)\right)\left(\sum_{j=1}^{N} A_{i, j} \beta_{\sigma_{j}}^{j} V_{j}(t)\right) .
$$

The different recovery and infection rates describe the heterogeneity of individuals in their usage of communication networks; and therefore their different impacts on the security level and virus spreading into this system. 
The SIS epidemic process has always had one stable stationary regime, the zero solution. But, in addition, under certain circumstances on $\delta_{i}$ and $\beta_{i}$, there exists another stationary regime called a metastable state in our SIS epidemic process described in the paper [13].

We focus on the stationary metastable regime, for which $\lim _{t \rightarrow \infty} \frac{d V_{i}(t)}{d t}=0$, we can simplify the NIMFA equation, with the new notation $\lim _{t \rightarrow \infty} V_{i}(t)=V_{i}^{\infty}$. The new expression of the NIMFA equation is as follows:

$$
0=\delta_{i} V_{i}^{\infty}(\sigma)+\left(1-V_{i}^{\infty}(\sigma)\right)\left(\sum_{j=1}^{N} \beta_{\sigma_{j}}^{j} A_{i, j} V_{j}^{\infty}(\sigma)\right)
$$

where $\sigma$ represents the decision vector of the nodes. As we want to find an individual strategy of protection, we can classify the nodes. So, considering equation (2), we have the following relation between the component of the infection vector probability:

$$
V_{i}^{\infty}(\sigma)=1-\frac{1}{1+\sum_{j=1}^{N} A_{i, j} \frac{\beta_{\sigma_{j}}^{j}}{\delta_{i}} V_{j}^{\infty}(\sigma)} .
$$

Consdiering this infection probability, we define the payoff function where $\sigma=$ $\left(\sigma_{1}, \ldots, \sigma_{i}, \ldots, \sigma_{N}\right)$ is the action chosen by $i$ player:

$$
C_{i}(\sigma)= \begin{cases}G_{i} & \text { if } \sigma_{i}=0, \\ H_{i} V_{i}^{\infty}(\sigma) & \text { otherwise when } \sigma_{i}=1,\end{cases}
$$

with $G_{i}$ is the anti-virus price for player $i$ and $H_{i}$ is the cost for recovery for player $i$.

\section{Existence of Nash Equilibrium and Algortihm}

Definition 3.1 (Potential Game [8]) $P$ is called a potential function if and only if $\forall i \in\{1, \ldots, N\}$ :

$$
C_{i}\left(\sigma_{i}^{1}, \sigma_{-i}^{1}\right)-C_{i}\left(\sigma_{i}^{0}, \sigma_{-i}^{0}\right)=P\left(\sigma_{i}^{1}, \sigma_{-i}^{1}\right)-P\left(\sigma_{i}^{0}, \sigma_{-i}^{0}\right) .
$$

In our game, the common strategy set is a binary set composed of only two actions $(A=\{0,1\})$.

Property 3.1 We consider the following function $P$ :

$$
\begin{aligned}
P:\{0,1\}^{N} & \longrightarrow \mathbb{R} \\
\left(\sigma_{1}, \ldots, \sigma_{N}\right) & \longmapsto \sum_{j=1}^{N}\left(G_{j}\left(1-\sigma_{j}\right)+H_{j} \sum_{l=0}^{\sigma_{j}} V_{j}\left(l, \sigma_{-j}\right)\right) .
\end{aligned}
$$

where $\sigma_{j}$ is the action of player $j$. This function is a potential for our game. 
We conclude the following [8]:

Property 3.2 There exists a pure Nash Equilibrium for our game.

In next section, we describe an algorithm to compute the pure Nash equilibrium.

\subsection{Algorithm}

We are not able to determine explicitly the Nash equilibrium strategy, more precisely, the number of players that decide to invest at equilibrium. Then, we determine an algorithm in order to compute it.

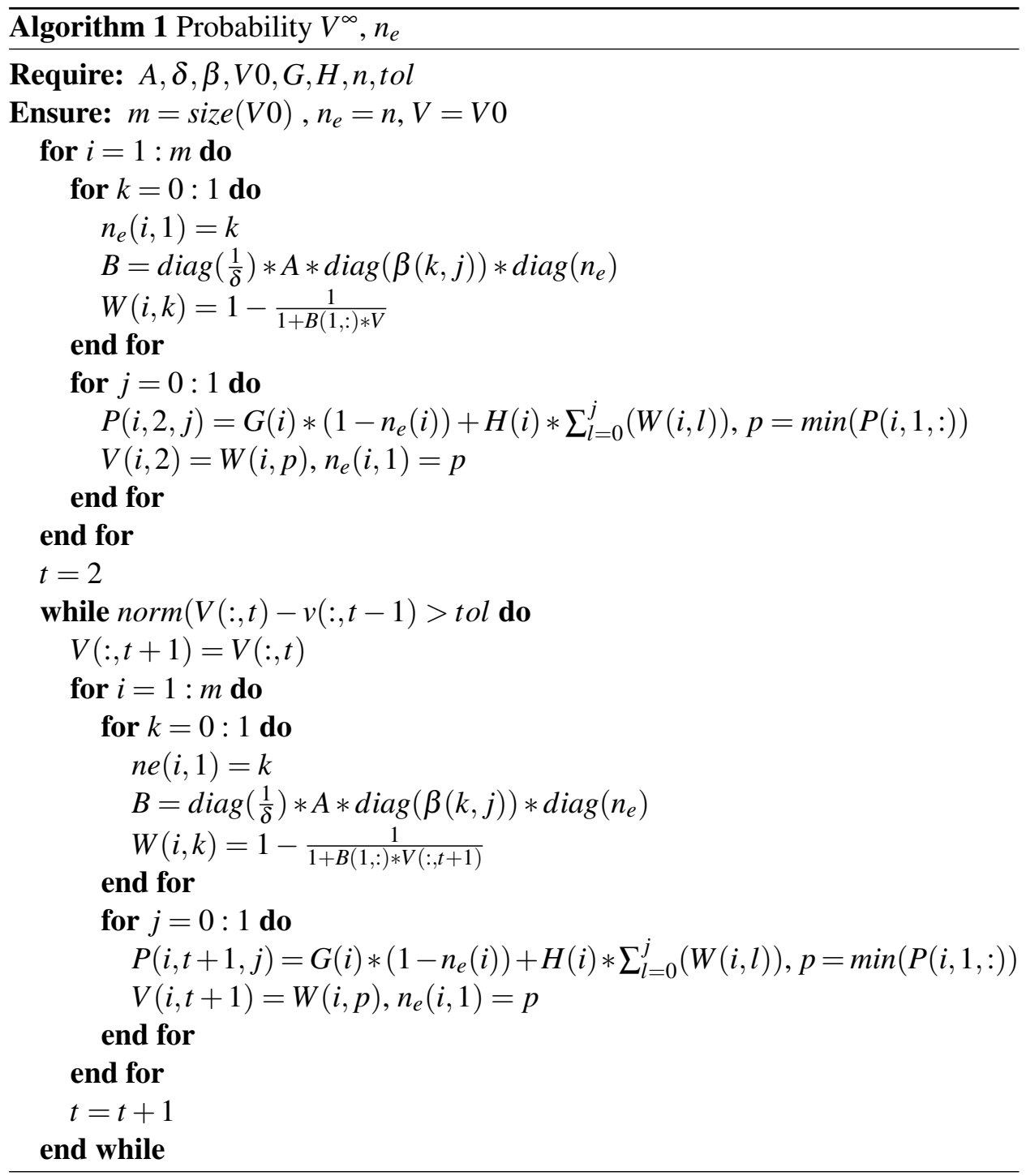

This algorithm is based on the two following features: 
- (i) A process of best response functions,

- (ii) A research of a fixed point solution.

In order to prove the convergence of the algorithm, we are going to use the following theorem for the best-response part of the algorithm.

Theorem 3.1 In a finite potential game, from any arbitrary initial outcome, the best response dynamics converge to a pure Nash Equilibrium.

Then, to prove the existence of a fixed point non-zero, we are refering to the theorem 1 and the lemma 2 in the paper [7]. We illustrate the performance of our algorithm in the section through simulations.

\section{Numerical Illustrations}

We run our algorithm on a specific network topology depicted on figure 1. In this topology, the graph is heterogeneous as nodes have different degrees. We define

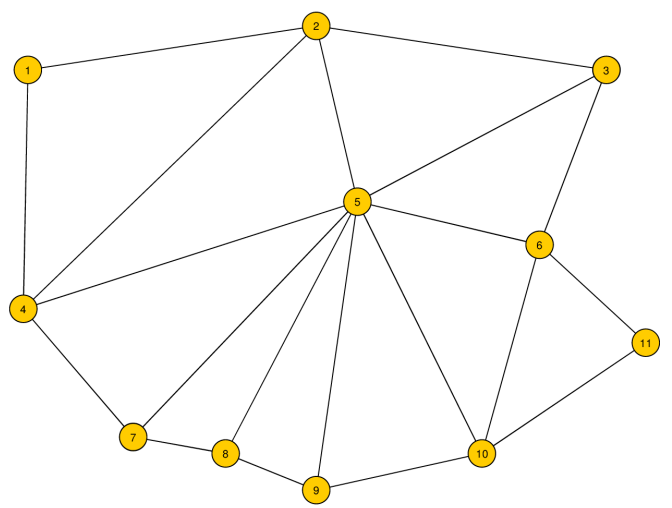

Figure 1: Incomplete graph 11 nodes

different scenarios depending on specific parameters of the model. For the first scenario, called the fully immunization game, we assume that when a node invests, its infection rate $\beta$ is equal to 0 , which could be viewed as an exclusion of the node from the network of infection.

\subsection{Fully immunization game}

In this part, the infection rate $\beta$ of a node is equal to zero if this node invests. This means the node does not participate into the virus spread. In this case, the convergence to a pure Nash equilibrium is obtained after very few iterations (around 10) and considering the following parameters: 
- $\delta=(0.52,1.34,1.59,1.68,0.75,0.64,0.62,0.72,0.69,0.58,0.61)$

- $\beta=(1.98,1.25,1.59,1.34,1.69,1.25,1.39,1.67,1.48,1.58,1.63)$

- $G=(51,52,50,51,50,52,53,51,48,47,49)$

- $H=(63,61,62,62,63,61,63,62,57,59,60)$

The Nash equilibrium obtained is given by the strategy vector

$$
N e=(1,1,1,1,0,1,1,1,1,0,1) .
$$

We observe, as expected, that node's degree has a non-negligeable influence on the strategic behavior. In fact, node 5, who has the highest degree in our topology and can be considered as a central node. Meanwhile, we observe that node 10 also decides to be protected and its degree is not the highest. By the way, node 10 has a higher contamination rate $\beta_{10}=1.58$ compared to node $6\left(\beta_{6}=1.25\right)$; and at the same time the recovery rate is lower for node 10 compared to node 6 . Therefore, we conclude that the topology and also the parameter of the virus dynamics influence the strategic behavior of the nodes and also the dynamical parameters of the virus.

\subsection{Healthy carrier game}

In this second scenario, we assume that the node can be an healthy carrier of the virus, in the sense that it does not suffer itself from the consequences but can contaminate healthy neighbors. In this scenario, there are two possible cases. The first one is that an investment induced that the node is more careless about its action in the network, and it takes more risk, increasing its contamination rate. The second hypothesis implies that each protected node still carries the virus. It is aware of the risk, and it is careful of its action in the network, decreasing the infection rate.

Hence, we do not talk about being infected anymore but being, a contaminated node is a carrier of the virus. This approach is similar to the Peltzmann theory[11]. Peltzmann introduces a notion called risk compensation or Peltzmann effect, which means that people behavior depends on the risk they take. Indeed, when the security belt was mandatory in the seventies, the number of car accident went up because people took more risk, thinking they were safe. In our context, we can expect the same behavior for computer usage. If an individual buys an anti-virus (a software or an application), and then feel protected, he may have a risky behavior and so its infection rate could be higher. Then, even if a device is protected, he can carry a virus or a malware that may use this device as a relay to propagate to other vulnerable devices. This point of view can be called the risky game.

In this context, we consider the following parameters of the model:

- $\delta=(0.52,1.34,1.59,1.68,0.75,0.64,0.62,0.72,0.69,0.58,0.61)$

- $\beta_{a}=(1.98,1.25,1.59,1.34,1.69,1.25,1.39,1.67,1.48,1.58,1.63)$ 


$$
\text { - } \beta_{n a}=(0.8,0.5,0.65,0.7,0.8,0.7,0.65,0.8,0.71,0.6,0.67)
$$

where $\beta_{a}$ (resp. $\beta_{n a}$ ) represents the vector of contamination rates of protected (resp. not protected) nodes. We observe that for each node, if he decides to protec itself, its contamination rate is higher. This property models the Peltzmann's effect. In this setting, the Nash equilibrium given by our algorithm proposed in previous section is

$$
N e=(0,0,0,0,0,0,0,1,1,0,1) .
$$

We observe that the Peltzman's effect impacts the behavior of the nodes and more nodes invest in the antivirus. This behavior is in accordance with the risky behavior induced by Peltzmann's assumption.

\subsection{Sustainable game}

We finally consider a setting in which a node is aware of the risk to boost the virus spread, and would be careful about its action. With this point of view, the game is called sustainable game. In this setting, we determine the following system parameters:

- $\delta=(0.752,0.94,0.59,0.68,0.75,0.94,0.82,0.92,0.99,0.78,0.91)$

- $\beta_{a}=(1.2,1.25,1.09,1.34,1.69,1.65,1.39,1.67,1.48,1.38,1.63)$

- $\beta_{n a}=(1.85,1.97,1.9,2.4,2.43,2.25,2.12,2.5,2.2,2.23,2.27)$

The Nash Equilibrium vector is

$$
N e=(0,0,0,0,0,0,0,1,1,0,0) .
$$

Only nodes 8 and 9 do not protect themselves, which is difficult to justify related to system parameters and topology. So, depending on the action of a node, its infection rate depends on the other node's decision. The different scenarios, which are modeled by a change of the infection rate depending on the action chosen, impacts the infection probabilities and the Nash equilibrium of the game. Whereas, it does not impact the existence of such equilibrium and the convergence of our algorithm. As aforementioned, the convergence of our algorithm is always valid. But, in the latter scenarios (healthy carrier and sustainable settings), we do not look at the risk to be infected, but the risk to carry the virus.

\section{Conclusion}

The identification of a potential for the virus protection game allows us to compute efficiently equilibria for arbitrary network topology and for a heterogeneous SIS dynamics. We have provided insights for scenarios like the Peltzmann's effect. In 
this case, we have shown the impact of the topology as well as the dynamics parameters on the outcome/equilibrium of the protection game.

Many perspectives can be considered following this work. We can plan to introduce stochasticity into the model and in the topology. In fact, realistic complex networks are stochastic in nature, meaning that links between nodes or relationships between individuals are generally dynamic and even stochastic. Other interesting features would be to consider a high level decision maker who can control system parameters like degrees (for example Facebook allows a maximum number of friends) or prices/costs. Considering this aspect, we could look for optimal control with equilibrium behavior of nodes as a constraint. New mathematical models and frameworks are therefore needed, taking into account this hierarchical structure of the system.

\section{References}

[1] James Aspnes, Navin Rustagi, and Jared Saia. Worm versus alert: Who wins in a battle for control of a large-scale network? In International Conference On Principles Of Distributed Systems, pages 443-456. Springer, 2007.

[2] Chris T Bauch. Imitation dynamics predict vaccinating behaviour. Proceedings of the Royal Society of London B: Biological Sciences, 272(1573):16691675, 2005.

[3] Chris T Bauch and David JD Earn. Vaccination and the theory of games. Proceedings of the National Academy of Sciences of the United States of America, 101(36):13391-13394, 2004.

[4] Yezekael Hayel, Stojan Trajanovski, Eitan Altman, Huijuan Wang, and Piet Van Mieghem. Complete game-theoretic characterization of sis epidemics protection strategies. In 53rd IEEE Conference on Decision and Control, pages 1179-1184. IEEE, 2014.

[5] Michael B Kelley. The stuxnet attack on irans nuclear plant wasfar more dangerous than previously thought. Business Insider, 20, 2013.

[6] MHR Khouzani, Saswati Sarkar, and Eitan Altman. Saddle-point strategies in malware attack. IEEE Journal on Selected Areas in Communications, 30(1):31-43, 2012.

[7] Piet Van Mieghem, Jasmina Omic, and Robert Kooij. Virus spread in networks. IEEE/ACM Transactions on Networking, 17(1):1-14, 2009.

[8] Dov Monderer and Lloyd S Shapley. Potential games. Games and economic behavior, 14(1):124-143, 1996. 
[9] William Hugh Murray. The application of epidemiology to computer viruses. Computers \& Security, 7(2):139-145, 1988.

[10] Jasmina Omic, Ariel Orda, and Piet Van Mieghem. Protecting against network infections: A game theoretic perspective. In INFOCOM. IEEE, 2009.

[11] Sam Peltzman. The effects of automobile safety regulation. The Journal of Political Economy, pages 677-725, 1975.

[12] P Van Mieghem and Ruud van de Bovenkamp. Accuracy criterion for the mean-field approximation in susceptible-infected-susceptible epidemics on networks. Physical Review E, 91(3):032812, 2015.

[13] Piet Van Mieghem and Jasmina Omic. In-homogeneous virus spread in networks. arXiv preprint arXiv:1306.2588, 2013. 